\title{
Degenerating the butterfly attractor in a plasma perturbation model using nonlinear controllers
}

\begin{abstract}
In this work, the dynamical behaviors of a low-dimensional model, which governs the interplay between a driver associated with pressure gradient and relaxation of instability due to magnetic field perturba- tions, are investigated. Besides that, two nonlinear controllers are constructed precisely to shift the equi- libria of the plasma model apart from each other. Simulation results show that shifting the equilibria can change the spacing of chaotic attractors, and subsequently break the butterfly wings into one or two symmetric pair of coexisting chaotic attractors. Furthermore, stretching the equilibria of the system apart enough from each other gives rise to degenerate the butterfly wings into several periodic orbits. In ad- dition, with appropriate initial conditions, the complex multistability behaviors including the coexistence of butterfly chaotic attractor with two point attractors, the coexistence of transient transition chaos with completely quasi-periodic behavior, and the coexistence of symmetric Hopf bifurcations are also observed.
\end{abstract}

Keyword: Multistability behaviors; Coexisting attractors; Shifting equilibria; Broken butterfly; Hopf bifurcation 